\title{
Gold Nanoparticle Plasmonic Superlattices as Surface Enhanced Raman Spectroscopy Substrates
}

Cristiano Matricardi, ${ }^{1, \&}$ Christoph Hanske, ${ }^{2, \&}$ Juan Luis Garcia-Pomar, ${ }^{1}$ Judith Langer, ${ }^{2}$ Agustín Mihi, ${ }^{1, *}$ and Luis M. Liz-Marzán ${ }^{2,3, *}$

${ }^{1}$ Institut de Ciència de Materials de Barcelona (ICMAB-CSIC), Campus de la UAB, 08193 Bellaterra, Catalonia, Spain

${ }^{2}$ CIC biomaGUNE and Ciber-BBN, Paseo de Miramón 182, 20014 Donostia San Sebastián, Spain

${ }^{3}$ Ikerbasque, Basque Foundation for Science, 48013 Bilbao, Spain

${ }^{\&}$ These authors contributed equally to this work

(*corresponding authors: amihi@icmab.es and llizmarzan@cicbiomagune.es)

KEYWORDS: SERS, gold nanoparticles, lattice plasmon, self-assembly, Rayleigh

anomaly

\begin{abstract}
Metal colloids are of great interest in the field of nanophotonics, mainly due to their morphology-dependent optical properties, but also because they are high quality building blocks for complex plasmonic architectures. Close-packed colloidal supercrystals not only serve for investigating the rich plasmonic resonances arising in strongly coupled arrangements, but also enables tailoring the optical response, on both the nano- and the macroscale. Bridging these vastly different length scales at reasonable fabrication costs has remained fundamentally challenging, but is essential for applications in sensing, photovoltaics or optoelectronics, among other fields. We present here a scalable approach to engineer plasmonic supercrystal arrays, based on the template-assisted assembly of gold nanospheres with topographically patterned polydimethylsiloxane molds. Regular square arrays of hexagonally packed supercrystals
\end{abstract}


were achieved, reaching periodicities down to $400 \mathrm{~nm}$ and feature sizes around $200 \mathrm{~nm}$, over areas up to $0.5 \mathrm{~cm}^{2}$. These two-dimensional supercrystals exhibit well-defined collective plasmon modes that can be tuned from the visible through the near-infrared by simple variation of the lattice parameter. We present electromagnetic modelling of the physical origin of the underlying hybrid modes, and demonstrate the application of superlattice arrays as surface-enhanced Raman scattering (SERS) spectroscopy substrates which can be tailored for a specific probe laser. We therefore investigated the influence of the lattice parameter, local degree of order, and cluster architecture, to identify the optimal configuration for highly efficient SERS of a non-resonant Raman probe with $785 \mathrm{~nm}$ excitation.

Ultrasensitive molecular detection and specific quantification of analytes have been leading motivations in the development of advanced spectroscopic techniques. ${ }^{1}$ Raman spectroscopy, which probes vibrational transitions, allows the label-free identification of a wide variety of molecules, thereby becoming a highly relevant tool in disciplines including biology, medicine, or forensics. ${ }^{2-4}$ However, the extremely low cross section of Raman scattering usually requires either large amounts of analyte or very powerful laser sources, which can in turn degrade the molecules. ${ }^{5}$ Surface-enhanced Raman scattering (SERS) has mitigated this issue, on the basis of the amplification of the Raman signal of molecules in contact with nanostructured metallic surfaces. ${ }^{6,7}$ The main mechanism behind the observed huge Raman signals is the electromagnetic enhancement, due to a local confinement of the electromagnetic field, which is especially strong within plasmonic hot spots between close-packed metal nanoparticles. $^{8}$ 
Consequently, colloidal mesostructures have a great potential as highly effective SERS substrates, ${ }^{9,10}$ whereby their performance depends sensitively on the interparticle distance. $^{11,12}$ Direct writing techniques like e-beam or focused ion beam lithography can be used to obtain nanoparticle arrangements with the nanometer-sized gaps necessary for SERS, but are very inefficient in terms of time and cost. ${ }^{13}$ Therefore, selfassembly of chemically synthesized plasmonic nanoparticles is often utilized for substrate preparation. Methods as simple as drop casting or precipitation of colloidal dispersions may yield assemblies that are able to sufficiently amplify Raman cross sections and enable even single molecule detection, ${ }^{14-16}$ which however usually comes at the cost of poor signal reproducibility. ${ }^{17,18}$ Strategies for preparing homogeneously structured nanoparticle films by template-assisted self-assembly have been improved significantly over the last decade, now having the potential to solve the above mentioned issues. ${ }^{19-22}$ These substrates featuring both a high density of hot spots and improved uniformity have been employed, for example, in the detection of gases like carbon monoxide or pyrene, ${ }^{23,24}$ as well as in monitoring the expression of bacterial quorum sensing molecules via SERS. ${ }^{25}$

The occurrence of collective plasmon resonances is directly connected to the presence of hot spots in metal nanoparticle assemblies, which often dominate the optical response of nanoparticle films. ${ }^{26-28}$ Near-field coupling between neighboring NPs can give rise to hybridized modes in clusters. These resonances are usually red-shifted and broadened compared to the localized surface plasmon resonances (LSPRs) of isolated particles. Besides single particle properties and gap size, the frequencies at which such collective resonances appear, depend on cluster size and symmetry. ${ }^{22,29}$ Further, the introduction of superordinate periodic patterns can give rise to additional diffractive resonances due to optical far-field interactions. These so-called lattice plasmon modes can be tuned 
over a wide frequency range by varying lattice parameters and have been reported to yield remarkably sharp resonances. ${ }^{21,28,30}$ Intriguingly, Reinhard et al. were able to demonstrate within micrometer-sized areas, the effect of the e-field enhancement of periodic nanoparticle cluster arrays on the intensity of the SERS signal. ${ }^{31,32}$ Taking into account the manifold factors determining the overall field enhancement in these assemblies, such as cluster dimensions, lattice parameters, number of layers, and the overall degree of order, a detailed investigation is required to fully understand and utilize the complex electromagnetic coupling modes within and between clusters. The ability of tuning the frequency of these hybrid plasmonic-photonic resonances further facilitates nanophotonic device engineering. ${ }^{33}$ Still, the challenge resides in fabricating hierarchical assemblies that operate at visible wavelengths and display sufficiently good homogeneity over macroscopic areas. ${ }^{34,35}$

We present in this work a scalable, template-assisted assembly technique, capable of arranging gold nanospheres (AuNSs) into regular, periodic arrays of well-defined plasmonic clusters over large areas. The obtained 2D superlattices exhibit both strong near-field coupling and an optical response that can be tuned from the visible through the near-infrared (NIR). Topographically patterned elastomeric molds featuring square arrays of submicrometer-sized holes were used to guide the assembly of monodisperse AuNSs into organized cluster arrays, over areas up to $0.5 \mathrm{~cm}^{2}$. The specific pattern on the polydimethylsiloxane (PDMS) mold determines the cluster diameter and lattice parameter, whereas the concentration of the AuNSs dispersion determines the number of particle layers in the clusters and the overall quality of the assembly. The distinct plasmonic-photonic hybrid resonances recorded by microspectroscopy can be precisely reproduced and explained by numerical simulations. These results allow us to tailor the optical response of the substrates for excitation with specific or available laser lines. In 
addition, we studied the correlation between the plasmon resonances sustained by the different geometrical assemblies and their performance as SERS substrates under 785 nm excitation of the Raman probe 4-acetamidothiophenol (4-AMTP). This laser line fits the first biological optical transparency window, thus providing enhanced light penetration in tissues, and is particularly interesting for biomolecular detection in complex media.

\section{Results and Discussion}

The monodisperse gold nanoparticles used in this work were stabilized with (PEG, 6kD) and featured an average diameter of $52 \mathrm{~nm}$ (standard deviation: $2 \mathrm{~nm}$ ), with a corresponding LSPR at $\lambda_{L S P R}=532 \mathrm{~nm}$ (Figure S1, SI). The use of patterned PDMS molds to fabricate nanopatterned SERS substrates, ${ }^{36}$ or to direct the assembly of gold colloids into ordered arrays, is an appealing approach due to both the intrinsic low cost of the technique and the large areas that can be attained. Successful template-assisted self-assembly of AuNSs has been previously reported into micrometer-sized square pyramids, ${ }^{37}$ leading to highly regular plasmonic supercrystals. This was, however, unsuitable to obtain structures exhibiting a strong optical response at visible wavelengths, for which the cluster arrays should feature order at the submicrometer scale. ${ }^{31}$ Such a structuration was achieved in the present work, by using PDMS molds featuring square array geometries with lattice parameters $(L)$ of 400, 500, 600, 740 and $1600 \mathrm{~nm}$, and hole diameters $(d)$ of 230, 270, 330, 440 and $960 \mathrm{~nm}$, respectively (see

\section{Methods).}




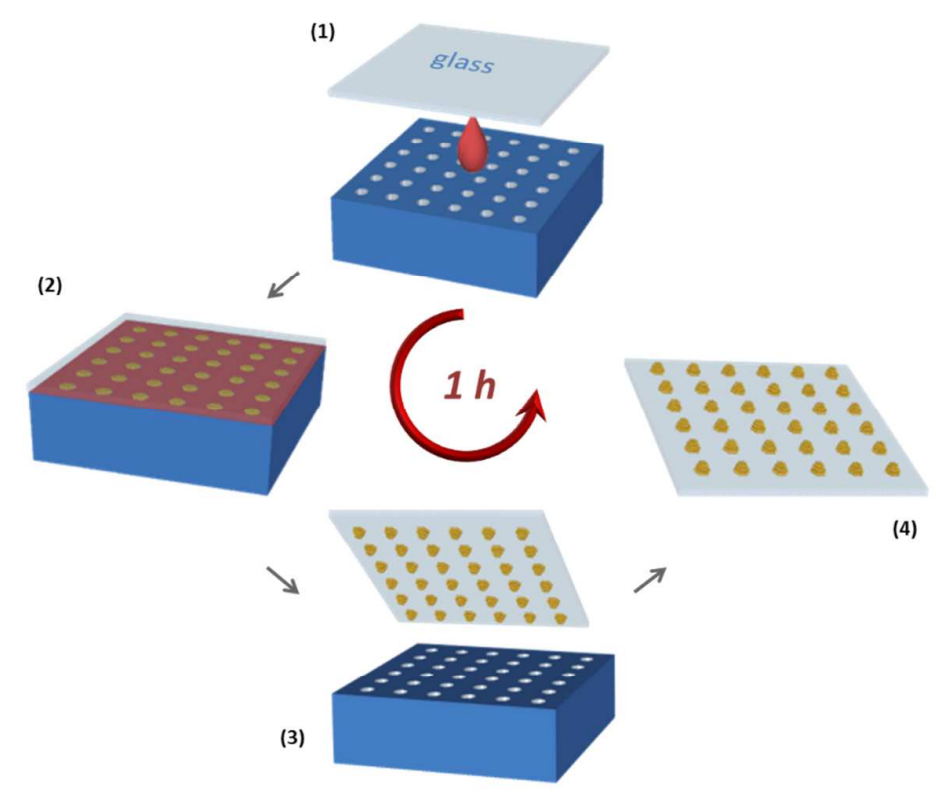

Figure 1. Schematic representation of the template-assisted assembly of gold nanospheres into hierarchical superlattices: (1) Deposition of an aqueous AuNSs dispersion on the surface of a patterned PDMS stamp. (2) A glass coverslip is placed on top of the PDMS, spreading the colloidal dispersion over the patterned surface. (3) Removal of the glass substrate carrying the assembled superlattice.

The process of template-assisted assembly of AuNSs into arrays of close-packed, mesoscopic clusters is depicted in Figure 1. First, a drop $(1 \mu \mathrm{L})$ of the AuNS colloidal dispersion (Figure 2a) is deposited on the surface of a PDMS mold (Figure 2b) patterned with square arrays of holes, with $L$ ranging from 400 to $1600 \mathrm{~nm}$ and similar filling factors. The filling factor $(F F)$, corresponds to the percentage of effective area that can be covered with nanoparticles and is defined as $F F=\frac{\pi R^{2}}{L^{2}}$, with $R$ being the radius of the holes, which yields $F F \sim 0.25-0.27$ for the employed stamp geometries. Second, a hydrophilized glass coverslip is placed on top of the PDMS surface, spreading the colloid into the PDMS features that direct the assembly. Finally, after about 1 hour, evaporation of the dispersion medium (water) is completed and the glass substrate carrying the assembled AuNSs can be carefully lifted off. The supercrystal films (Figure 2c) display iridescent colors under white light illumination, showing an 
angular dependence similar to that of the corresponding molds. Scanning electron microscopy (SEM) images (Figure 2 d-f) from representative samples evidence the long-range order of the AuNS clusters on glass. It is worth noting that the AuNSs pack hexagonally within the clusters, and that even for a fixed hole size the width and height of individual assemblies can vary slightly.

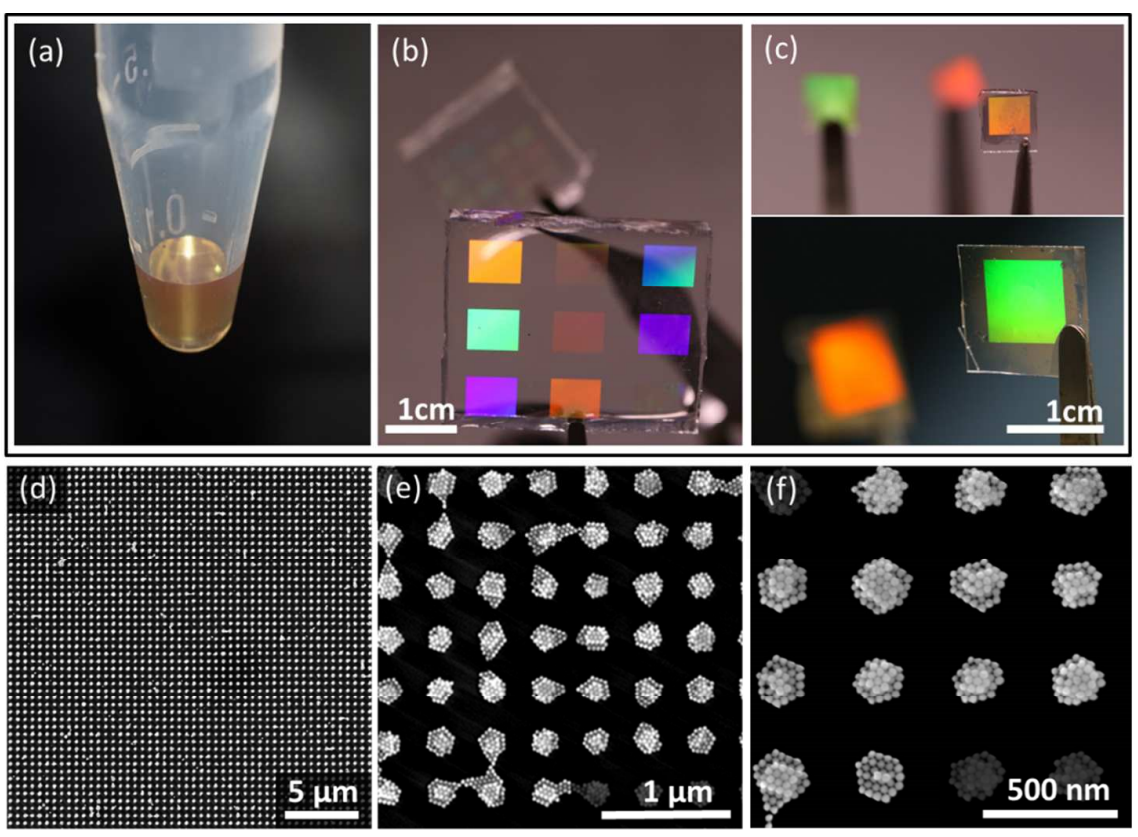

Figure 2. Upper panel: Photographs of a dispersion of AuNSs (a), the PDMS molds used for the assembly (b), and AuNS assemblies obtained on glass (c). Lower panel: SEM micrographs of representative square lattice AuNS clusters, at different magnifications (d-f).

The efficiency of AuNS assemblies for sensing is largely determined by their optical response. Therefore, we optimized the templated assembly conditions, guided by optical measurements (extinction $=1-\mathrm{R}-\mathrm{T}$, where $\mathrm{R}$ and $\mathrm{T}$ correspond to Reflectance and Transmittance spectra from the films) and SEM inspection. We thus prepared assemblies with varying AuNS concentrations (see Figure S2, SI), concluding that the average number of particles per cluster could be adjusted via the AuNS concentration and had a pronounced influence on the optical quality of the substrates. Combined 
inspection of the topology and the extinction spectra indicated that ordered assemblies with well-defined plasmon resonances were obtained for intermediate $\mathrm{Au}$ concentrations (33- $40 \mathrm{mM})$. Lower concentrations resulted in incomplete clusters with broad resonances, whereas higher concentrations produced accumulation or bridging with additional AuNSs between clusters, accompanied by an increased optical background.

The influence of the array periodicity on the optical response was studied using assemblies with lattice parameters ranging from 400 to $1600 \mathrm{~nm}$, made from dispersions with optimized gold concentration. Representative SEM images and extinction spectra of the resulting cluster arrays are displayed in Figure 3. From inspection of the optical spectra, two main regions can be identified: the first region at lower wavelengths shows a local extinction maximum at $\lambda \sim 560 \mathrm{~nm}$. This peak is close to the dipolar mode of a single AuNS $\left(\lambda_{L S P R}=532 \mathrm{~nm}\right.$ in water $)$ and is likely influenced by higher order coupling modes of the clusters, which are typically observed for interparticle distances smaller than $2 \mathrm{~nm}^{28,38}$ When the NSs within the clusters are closer to each other, a stronger degree of plasmon coupling can be expected, and higher order cluster modes appear at energies close to the single particle LSPR. These higher order cluster modes, which have been discussed in previous works, ${ }^{28,38}$ can shift the peak to $\lambda \sim 560 \mathrm{~nm} .{ }^{22,39}$ The second region at higher wavelengths displays the most intense extinction peak and clearly red-shifts when increasing the lattice parameter of the assembly. The strong correlation of the latter extinction peak with the lattice parameter indicates that this resonance originates from the hybridization of the plasmonic cluster modes with the diffractive lattice mode. 


\section{(a)}

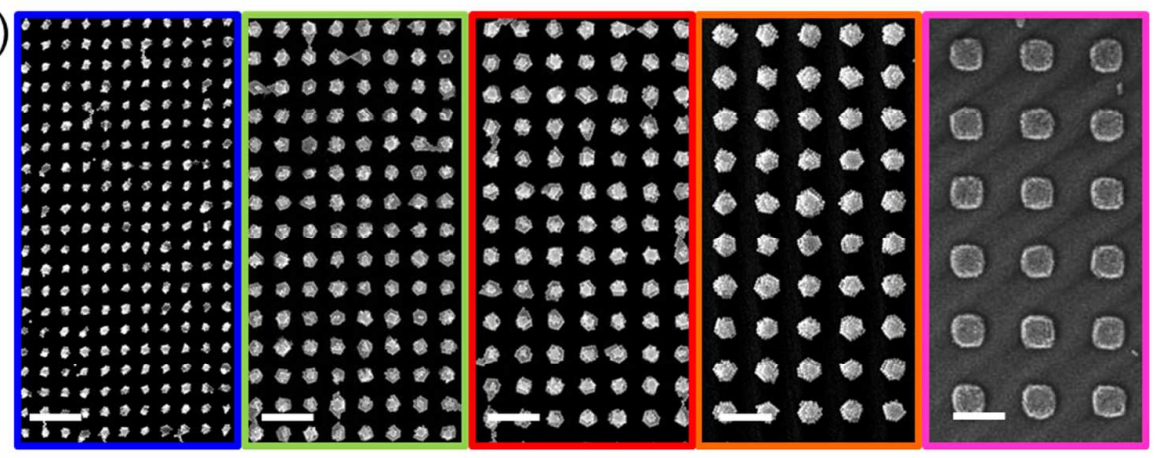

(b)

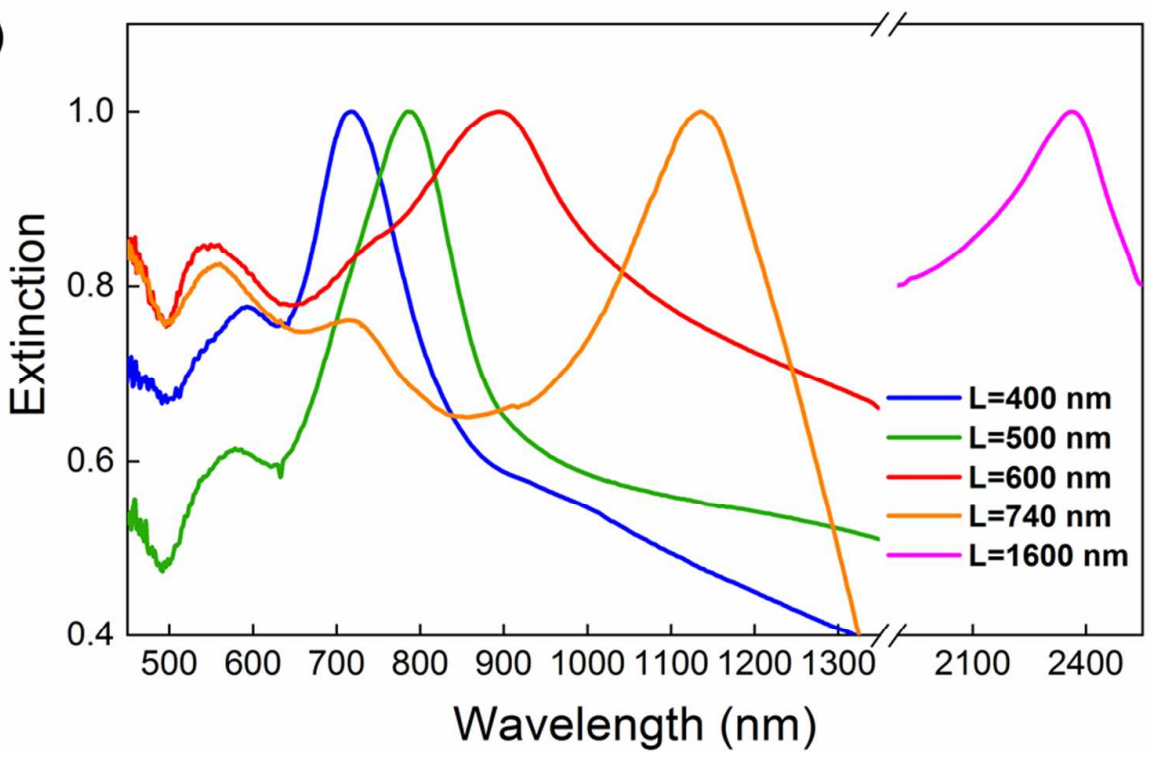

Figure 3. Structural and optical characterization of supercrystal assemblies with varying lattice parameter $L$. (a) SEM micrographs of gold nanoparticle arrays with different lattice parameters: from left to right $L=400 \mathrm{~nm}, L=500 \mathrm{~nm}, L=600 \mathrm{~nm}, L=740 \mathrm{~nm}$, and $L=1600 \mathrm{~nm}$. (b) Extinction spectra, normalized to the maximum, of AuNS cluster arrays with different lattice parameters (see labels). Scale bar: $1 \mu \mathrm{m}$.

These resonances, launched by light diffracted by the grating, are typically called surface lattice resonances, ${ }^{40}$ and are usually observed near a Rayleigh anomaly, i.e., a change in the magnitude of the reflectivity associated with the onset of diffraction from the grating. ${ }^{41}$ Rayleigh anomalies are grazing waves propagating in the plane of the array. In our system, the electromagnetic field tends to concentrate in the regions with the highest refractive index. As a consequence, the light scattered at the Rayleigh anomaly propagates at the glass, and hybridization of the collective surface plasmon 
resonances inside the AuNS clusters with the lattice modes is dominated by the refractive index of the substrate (Figure S4). ${ }^{42,43}$ This implies that the wavelength position of the lattice modes can be predicted via a simple formula, corresponding to the first order of diffraction, $\lambda_{\max } \simeq L n_{\text {glass }}$, where $n_{\text {glass }}$ is the refractive index of the glass substrate. This formula fits very accurately with the positions of the maxima observed for different lattice parameters $L$, as shown in Figure 3b.

(a)

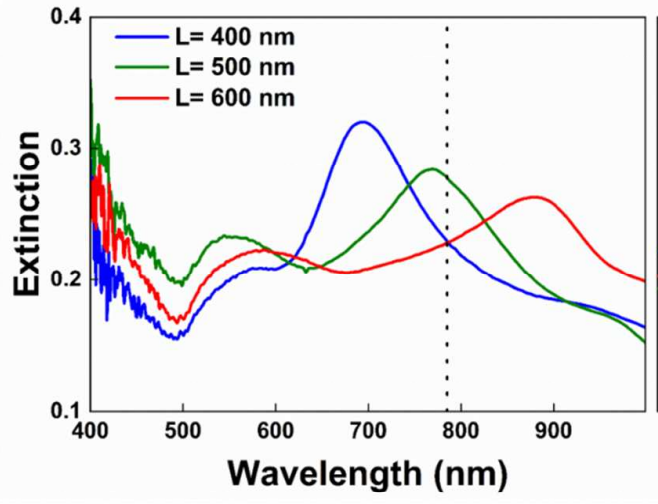

(c)

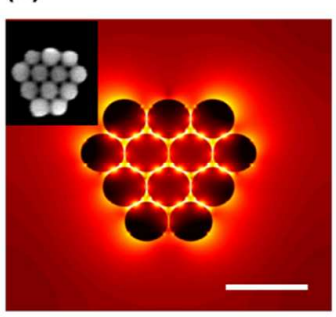

(b)

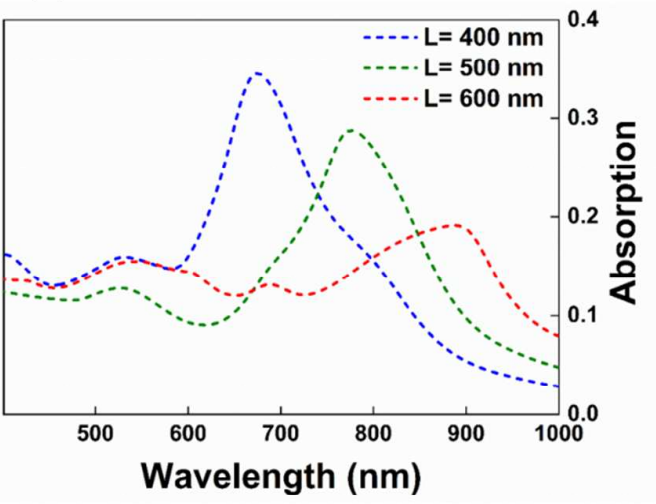

(e)
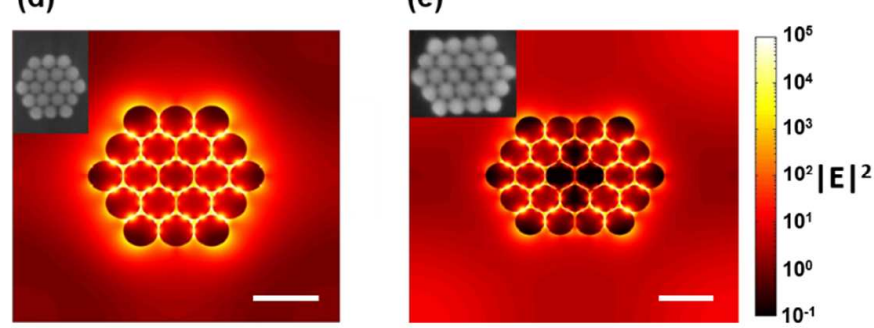

Figure 4. (a) Extinction spectra measured from AuNS arrays for varying lattice parameter $L$. The black dashed line marks the excitation laser wavelength used for surface-enhanced Raman spectroscopy, $\lambda=785 \mathrm{~nm}$. (b) Absorption spectra from FDTD simulations. (c,d,e) Snapshots of a transversal cut of an AuNS superlattice unit cluster (insets are SEM micrographs with analogous configuration), showing the intensity of the electric field $|E|^{2}$, for lattice parameters $L=400 \mathrm{~nm}, 500 \mathrm{~nm}$, and $600 \mathrm{~nm}$, calculated at an excitation wavelength of $785 \mathrm{~nm}$. Scale bars represent $100 \mathrm{~nm}$.

Further insight into the physical origin of the hybridized lattice resonances is gained by comparing the measured extinction spectra with the simulated absorption of the 
corresponding periodic structures, calculated by Finite Difference Time Domain simulations (FDTD). This comparison is displayed in Figure 4a,b for assemblies with $L=400,500$, and $600 \mathrm{~nm}$, all having similar filling factors of AuNS $(F F=0.25-0.27)$, which correspond to maximum particle densities of 75,76 and $83 \mathrm{AuNS} / \mu^{2}$, respectively. The values of AuNSs per unit area were calculated by inspection of SEM micrographs (Table S1). Corresponding SEM images of each model cluster type, with similar nanoparticle density, are included as insets in Figure 4c,d,e. As exemplified in the Supporting Information (Figure S3), the cluster size was found to influence the position of the optical resonance only marginally, for assemblies containing 9 or more particles. Still, variations in the configuration and orientation of the clusters lead to a slight damping of the extinction intensity and an increase of the full-width-at-halfmaximum (FWHM) in the experimental data, as compared to numerical simulations of periodically arranged, identical clusters. Aside from that, we find excellent agreement between the measured and the calculated spectra.

In general, the spectral position of the plasmon resonances is expected to correlate with the excitation of the highest local field factor and to affect the maximum Raman signal enhancement. ${ }^{8}$ This should allow us to engineer the optical properties of the AuNS assemblies in a straightforward manner, toward targeting excitation with a specific laser line. Herein, we focus on irradiation with a laser of $785 \mathrm{~nm}$ wavelength, which is often preferred for optical investigations of biological samples as it matches the first optical transparency window. Comparing the extinction spectra of supercrystal films with different geometries (Figure 4a,b), we find that arrays with lattice parameter $L=500 \mathrm{~nm}$ have their main resonance band close to $785 \mathrm{~nm}$. Snapshots of the electric field intensity $|E|^{2}$ at a transversal section of the periodic AuNS cluster arrays (Figure 4c-e) also display the highest enhancement for the lattice with $L=500 \mathrm{~nm}$, under excitation at 785 
nm. This extra field enhancement originates from the periodic arrangement of the plasmonic clusters in a square lattice, which becomes obvious when comparing the field enhancement profiles of isolated and periodically arranged clusters (Figure S5, SI). Average enhancement factor spectra, calculated (see Methods) by averaging the surface integral of the field enhancement factor $\frac{|E|^{4}}{\left|E_{0}\right|^{4}}$ over the whole surface of the available gold nanospheres, where $E_{0}$ is the incident electric field, further corroborate the latticedependent enhancement effect and again predict the strongest fields for $L=500 \mathrm{~nm}$ (Figure S6, SI). ${ }^{44}$

To verify this analysis experimentally, we further investigated the performance of different cluster arrays as SERS substrates under $785 \mathrm{~nm}$ excitation, using 4acetamidothiophenol (4-AMTP) as a model probe. A representative SERS spectrum of 4-AMTP is shown as an inset in Figure 5, revealing the characteristic main vibrational modes at $1081 \mathrm{~cm}^{-1}$ (C-S and ring stretch) and at $1591 \mathrm{~cm}^{-1}$ (ring stretch). ${ }^{45}$ In order to assess to what extent the lattice resonance mode influences the SERS signal of the substrates, we compared different monolayered cluster arrays with $L=400,500,600$, and $740 \mathrm{~nm}$. Whereas the lattice resonances of the largest arrays are far away from the spectral region of interest, we observed that for this geometry, clusters with up to three AuNS layers can be obtained due to the increased depth of the holes in the mold (Figure S7), which allowed us to study also the influence of cluster height on the SERS enhancement.

We explored the local homogeneity of the samples, by recording 100 individual data points over an area of $10 \times 10 \mu \mathrm{m}^{2}$ and averaging for each measurement. As the SERS effect directly mirrors the near-field generated at the nanometer scale, even subtle changes in the local NP order, as well as the number and geometry of hot spots, can 
cause significant intensity fluctuations. Additionally, the 3D packing of AuNS into multilayered structures is known to affect the SERS response. ${ }^{27}$ Therefore, a precise correlation of the local array morphology with the recorded signal enhancement was ensured through SEM characterization of the sites selected for SERS measurements. The results are summarized in Figure 5, where the baseline-corrected average intensity of the $1081 \mathrm{~cm}^{-1}$ vibration is plotted as a function of the lattice parameter, for a typical set of measurements. For superlattices consisting of monolayer clusters (green triangles), a decrease of $L$ from 740 to $500 \mathrm{~nm}$ leads to a nearly linear signal increase by over $200 \%$, followed by a slight drop at $L=400 \mathrm{~nm}$, in agreement with the predictions from FDTD simulations. The sample with $L=740 \mathrm{~nm}$ further exemplifies how the extension from 2D to 3D assembly affects the SERS response: with the transition from monolayer to double layer clusters (red circle) the average SERS signal rises by more than $90 \%$, whereas a third layer (blue square) leads to a further signal enhancement of only $30 \%$. We attribute the higher counts to an increased hot spot density due to the $3 \mathrm{D}$ packing. A detailed characterization of the probed arrays, as well as a plot of the SERS intensity normalized by the surface density of particles, is provided in the Supporting Information (Figure S8, SI).

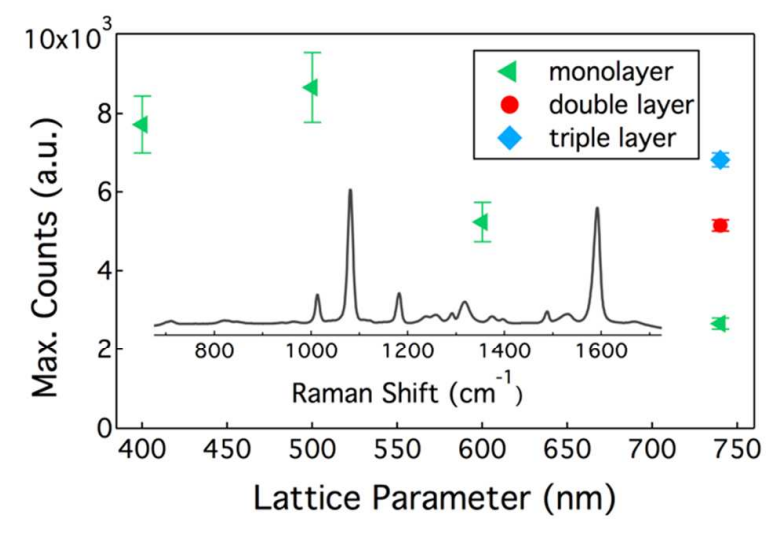

Figure 5. SERS signal at $1081 \mathrm{~cm}^{-1}$, as a function of the lattice parameter. The maximum SERS intensity was found for $L=500 \mathrm{~nm}$, in agreement with the good spectral 
match between the lattice plasmon wavelength and the $785 \mathrm{~nm}$ excitation laser, whereas the resonances of arrays with shorter and longer lattice parameters are detuned from the excitation laser (see Figure 4). The error bars depict the standard deviation of the signal in the probed $10 \times 10 \mu \mathrm{m}^{2}$ areas. The inset spectrum was recorded on a sample with $L=$ $500 \mathrm{~nm}$.

Overall, samples with an optical resonance close to the excitation wavelength of the laser exhibited significantly higher SERS enhancement than detuned samples. Intriguingly, monolayer samples tailored to the excitation wavelength ( $L=500 \mathrm{~nm})$ yielded a total signal, even higher than that from non-optimized multilayer arrays containing nearly three times as many particles $(L=740 \mathrm{~nm})$. It should be noted that for arrays with small lattice parameters the average local SERS signals typically fluctuated more (10-20\% standard deviation for $L=400$ and $500 \mathrm{~nm})$ than for assemblies with large lattice parameters $(2-5 \%$ standard deviation for $L=600$ and $740 \mathrm{~nm})$. Interestingly, probing monolayer arrays of varying quality with the same lattice parameter $(L=500$ $\mathrm{nm}$ ), revealed a clear correlation between the standard deviation and the average SERS signal. An example is displayed in Figure 6a, showing that ordered arrays (stdev $10 \%$ ) reached up to 5 times higher enhancement than irregular ones ( $\operatorname{stdev} 35 \%$ ). SEM inspection further confirmed that both homogeneity and signal intensity are strongly correlated to the average cluster size, for a given $L$. Plotting the normalized signal at $1081 \mathrm{~cm}^{-1}$ as a function of the average cluster size revealed that the pronounced increase in SERS enhancement is not exclusively due to an increased number of hot spots. As can be seen in Figure $\mathbf{6 b}$, the counts per particle (i.e., per hot spot) remained nearly constant below a threshold value of 15, above which further enhancement was registered until reaching the geometrically determined maximum cluster size of 19 particles. The signal per particle achieved with full cluster arrays (i.e., 19 particles for $L=500 \mathrm{~nm}$ ) was at least 3 times higher than that for a random sub-monolayer, obtained 
using the same method but a non-structured stamp. These observations are in agreement with FDTD simulations of different cluster arrays with $L=500 \mathrm{~nm}$, predicting both a cluster size-dependent hybridization efficiency (Figure S3) and stronger near-field enhancement in periodic arrays, as compared to isolated clusters (Figure S5, SI). A certain scatter of the counts per particle observed for clusters of identical size can be attributed to local structural defects: as illustrated in Figure 6c, comparing two arrays with identical lattice parameter and average cluster diameter, the enhancement clearly depends on the degree of order within the assembly, i.e., on the strength of the lattice resonance. Additional information on the homogeneity and reproducibility of the films is provided in the Supporting Information (Section 10).

$(\mathrm{a})$

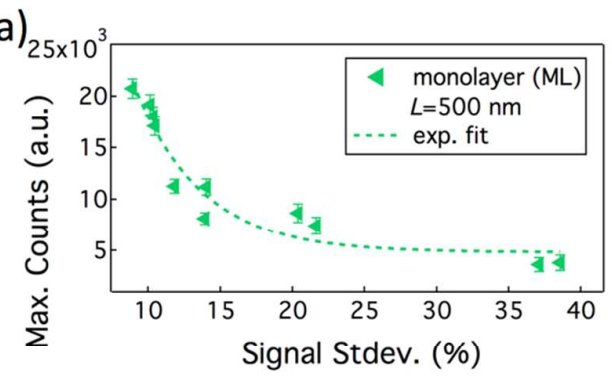

(b)
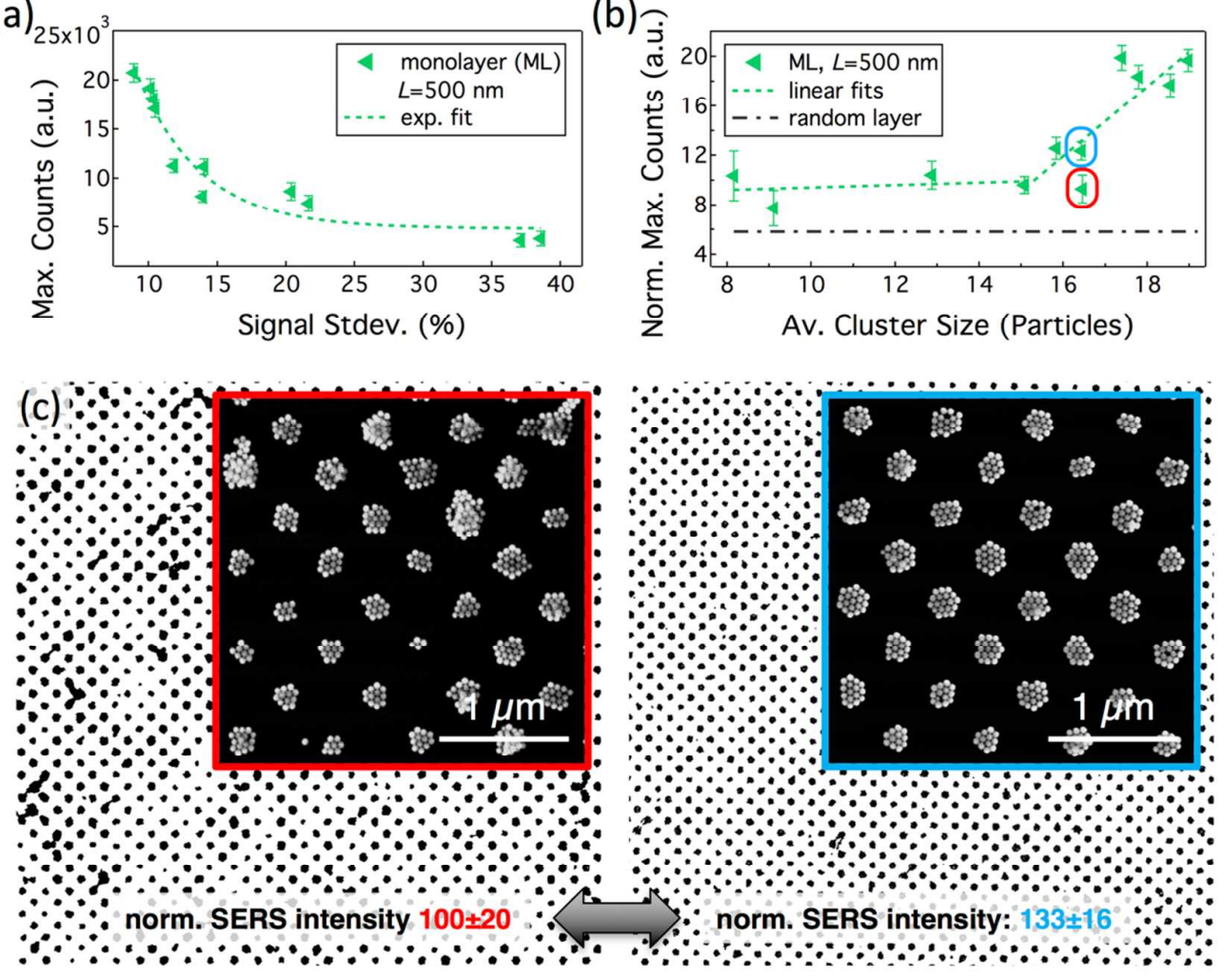

Figure 6. Correlated SERS and SEM characterization of monolayer arrays with fixed $L=500 \mathrm{~nm}$ and varying degree of assembly quality. (a) Increasing inhomogeneity, represented by the standard deviation (Stdev) of counts at $1081 \mathrm{~cm}^{-1}$, was accompanied by an overall decrease in signal strength. (b) The signal per particle was nearly constant for clusters smaller than 15 particles, but increased for larger clusters. The grey, dashed 
line depicts the signal of a random monolayer (see Figures S8,S9 for details). (c) For clusters of identical average diameter (marked by red and blue circles in b), the normalized signal depends strongly on the order within the array, illustrated with binary and inset grayscale SEM images. The error bars in $(a, b)$ correspond to the standard deviation of the signal within the probed $10 \times 10 \mu \mathrm{m}^{2}$ areas.

\section{Conclusions}

We have demonstrated the organization of gold nanospheres into square array supercrystals with different lattice parameters, from 400 to $1600 \mathrm{~nm}$, by using patterned PDMS molds as templates. The obtained 2D supercrystals exhibited well-defined resonance bands in the extinction spectra, which were tunable throughout the visible and NIR ranges, as a function of the lattice parameter, and were accurately reproduced by FDTD simulations. Theoretical modeling confirmed that the resonances originate from the hybridization of photonic lattice modes with plasmonic resonances sustained by individual AuNP clusters. The presence of such lattice plasmons guided the fabrication of supercrystal films with resonances targeting specific laser lines. We additionally explored the performance of 2D cluster arrays as SERS substrates, using 4AMTP as a model Raman probe. Correlation of Raman and SEM measurements demonstrated that, lattices with a periodicity $L=500 \mathrm{~nm}$ were the most effective SERS platforms under $785 \mathrm{~nm}$ laser excitation, in agreement with the predictions by a simple model based on extinction. The signal intensity was also found to strongly depend on the degree of order within the arrays, and, intriguingly, monolayer clusters with the optimal lattice parameter may even display larger enhancement than non-optimized multilayered assemblies. Investigation of $L=500 \mathrm{~nm}$ monolayer arrays of different qualities indicated that the SERS intensity per particle grows linearly with the particle number, for clusters containing more than 15 particles, eventually reaching over three- 
fold enhancement as compared to non-resonant random assemblies. This process thus leads the way toward highly efficient, uniform and reproducible SERS substrates.

\section{Methods}

Materials. Hexadecyltrimethylammonium chloride (CTAC, 25\% W/W), hydrogen tetrachloroaurate trihydrate $\left(\mathrm{HAuCl}_{4} \cdot 3 \mathrm{H}_{2} \mathrm{O}, \geq 99.9 \%\right)$, L-ascorbic acid $(\geq 99 \%)$, sodium hypochlorite solution (10-15\% available chlorine), sodium borohydride $\left(\mathrm{NaBH}_{4}, 99 \%\right)$, poly(ethylene glycol) methyl ether thiol (PEG-6K-SH, $\mathrm{M}_{\mathrm{n}}=6,000 \mathrm{~g} / \mathrm{mol}$ ), and 4acetamidothiophenol were purchased from Sigma-Aldrich and employed without further purification. Polydimethylsiloxane $\left(\right.$ Sylgard $^{\circledR}$ 184) was bought from Dow Corning. Water purified with a Milli- $Q^{\circledR}$ system was used in all experiments.

Nanoparticles synthesis. Monodisperse spherical gold nanoparticles with an average diameter of $52 \mathrm{~nm}$ (standard deviation: $2 \mathrm{~nm}$ ) were synthesized and characterized as previously published. ${ }^{37}$ In brief, a protocol that combines seeded growth with controlled particle etching was used. Initial seeds were prepared by adding $50 \mu \mathrm{L}$ of a $0.05 \mathrm{M}$ $\mathrm{HAuCl}_{4}$ solution to $5 \mathrm{~mL}$ of a $100 \mathrm{mM} \mathrm{CTAC}$ solution and injecting $200 \mu \mathrm{L}$ of a fresh $\mathrm{NaBH}_{4}(20 \mathrm{mM})$ solution while stirring vigorously. The resulting colloidal suspension was diluted by a factor of 10 with $100 \mathrm{mM}$ CTAC solution. These initial seeds were grown to $10 \mathrm{~nm}$ diameter by mixing $900 \mu \mathrm{L}$ of the diluted gold colloid with $10 \mathrm{~mL}$ of $25 \mathrm{mM}$ CTAC solution and $40 \mu \mathrm{L}$ of $100 \mathrm{mM}$ ascorbic acid, followed by injection of 50 $\mu \mathrm{L}$ of a $50 \mathrm{mM} \mathrm{HAuCl}$ solution under rapid stirring. Growth to the final size was achieved by diluting $250 \mu \mathrm{L}$ of the obtained suspension with $100 \mathrm{~mL}$ of a $25 \mathrm{mM}$ CTAC solution, adding $400 \mu \mathrm{L}$ of $100 \mathrm{mM}$ ascorbic acid, and injecting $500 \mu \mathrm{L}$ of a 50 $\mathrm{mM} \mathrm{HAuCl}_{4}$ solution under rapid stirring. After $1 \mathrm{~h}, 100 \mu \mathrm{L}$ of a dilute sodium hypochlorite solution (1-1.5 wt\% of available chlorine) were added stirring vigorously. 
After $5 \mathrm{~min}, 25 \mu \mathrm{L}$ of a $50 \mathrm{mM} \mathrm{HAuCl}_{4}$ solution were added and the reaction was left at $30{ }^{\circ} \mathrm{C}$ until a constant absorption at $400 \mathrm{~nm}\left(\mathrm{Abs}_{400}\right)$ was reached. The particles were then cleaned immediately by centrifuge washing twice at $3500 \mathrm{rpm}$ with $2 \mathrm{mM} \mathrm{CTAC}$ solution. PEGylation was typically done by setting the particles up to an $\mathrm{Au}^{0}$ concentration of $10 \mathrm{mM}$ (according to $\mathrm{Abs}_{400},{ }^{46,47}$ ) in $1 \mathrm{mM} \mathrm{CTAC}$ solution and adding $1 \mathrm{mg}$ of solid PEG-6k-SH per mL dispersion. The ligand exchange took place overnight and was followed by centrifuge washing with $300 \mu \mathrm{M}$ CTAC three times. Thereby, the particle concentration was increased up to $800 \mathrm{mM}$ to create a stock dispersion.

Nanoparticle assembly. For templated assembly, aliquots of the stock dispersion were diluted with suitable CTAC solutions to reach a final surfactant concentration of $50 \mu \mathrm{M}$ and $\mathrm{Au}^{0}$ concentrations between 13 and $90 \mathrm{mM}$, as denoted in the text. Borosilicate microscope coverslips (Menzel $\left.{ }^{\mathrm{TM}}, \# 1.5\right)$ cut into pieces of $8 \times 4 \mathrm{~mm}^{2}$ or $12 \times 12 \mathrm{~mm}^{2}$ were used as substrates, depending on the size of the PDMS mold. The substrate preparation protocol comprised cleaning with Hellmanex ${ }^{\mathrm{TM}}$ III solution, sonication in isopropanol, rinsing with water, and subsequent hydrophilization in a $\mathrm{UV}-\mathrm{O}_{3}$ chamber (ProCleaner ${ }^{\mathrm{TM}}$ ) for at least $45 \mathrm{~min}$. To fabricate periodically arranged plasmonic supercrystals, $1 \mu \mathrm{L}$ droplets of PEGylated AuNS dispersion were placed onto structured soft PDMS molds and covered with a hydrophilized glass substrate. After drying, the substrates were removed and the PDMS molds cleaned for reuse with adhesive tape, followed by rinsing with ethanol and water.

Soft molds fabrication. Soft PDMS molds were fabricated by pouring a 10:1 mixture of prepolymer and curing agent onto patterned silicon masters or their negative replicas with OrmoStamp ${ }^{\circledR}$ (Microresist Technology). ${ }^{48,49}$ The mixtures were degassed for 2 
hours to increase the percolation of the polymer inside the nanostructures and then cured for $45 \mathrm{~min}$ at $100{ }^{\circ} \mathrm{C}$. The PDMS molds were characterized by atomic force microscopy (see Figure S7, SI).

UV-vis measurements. Extinction spectra of dispersed AuNSs were recorded with an Agilent 8453 UV-vis spectrophotometer, using polystyrene cuvettes.

FTIR measurements. An FTIR spectrometer (Vertex 70 Bruker) attached to an optical microscope (Hyperion, Bruker) was used for far-field reflection and transmission measurements in the 400 to $3000 \mathrm{~nm}$ range. The background reflection spectrum was set with a silver mirror with $96 \%$ reflectivity in the tested range of wavelengths and the transmission background without sample. Both reflection and transmission spectra were collected with a $4 \mathrm{X}$ objective with a numerical aperture (N.A.) of 0.10 and a spatial mask. The spot size was fixed at $600 \times 600 \mu \mathrm{m}^{2}$.

Scanning electron microscopy. SEM images were recorded without prior sputtering using a FEI Quanta 250 microscope operated in low vacuum mode with an acceleration voltage of $10 \mathrm{kV}$ and a working distance of $5 \mathrm{~mm}$. The local particle densities were evaluated after suitable contrast and bright adjustments using the particle counting functionality of ImageJ. The arrays were categorized, either by manually counting the number of layered clusters or, where applicable, by comparing the cluster count and occupied area in overexposed (yields bottom layer) and underexposed (yields top layer) images. Thereby, structures with more than 1 particle sitting on top of a 2D cluster were registered as layered, and regions with less than $30 \%$ double layer content were defined as monolayer arrays. 
Transmission electron microscopy. TEM images were recorded with a JEOL JEM1400PLUS at an acceleration voltage of $120 \mathrm{kV}$. The particles were deposited from dilute dispersion on carbon-coated 400 square mesh copper TEM grids.

Numerical simulations. Numerical calculations were performed using Lumerical FDTD solutions (www.lumerical.com). The structural parameters of AuNS clusters for modelling were set according to the size measured from TEM images with an interparticle distance of $1 \mathrm{~nm}$. The dispersion model of gold was taken from Johnson and Christy $^{50}$ and the glass substrate was considered a medium with dispersive refractive index around $n_{\text {glass }} \sim 1.54-1.51$ at $400 \mathrm{~nm}$ and $1000 \mathrm{~nm}$, respectively. An unpolarized plane wave source impinging at normal incidence to different periodic AuNS clusters was modelled with a wavelength ranging from $400 \mathrm{~nm}$ to $2000 \mathrm{~nm}$, providing a good agreement with experimental results. A nonuniform FDTD mesh method was adapted to save computation resources and calculation time. Enhancement factors have been calculated measuring the surface integral of the electric field enhancement on the surface of the gold nanospheres and normalized by the total number of particles per $\mu \mathrm{m}^{2}$.

$$
\frac{\iint \frac{|E|^{4}}{\left|E_{0}\right|^{4}} d S}{\iint d S}
$$

Surface-enhanced Raman scattering (SERS) spectroscopy. An optimized cleaning procedure was necessary prior to the SERS measurements. To this end, the nanoparticle assemblies were treated first with oxygen plasma (Diener PICO, 0.4 mbar $\mathrm{O}_{2}, 200 \mathrm{~W}, 1$ min) and then exposed to $\mathrm{UV}-\mathrm{O}_{3}$ treatment for $30 \mathrm{~min}$. Immediately after cleaning, the substrates were incubated in $800 \mu \mathrm{L}$ of a freshly prepared $0.1 \mathrm{mM}$ aqueous 4-AMTP solution for at least $60 \mathrm{~min}$. Next, the samples were intensively rinsed with water to 
remove unbound molecules and dried in a $\mathrm{N}_{2}$ stream. SERS measurements were carried out in a confocal Raman microscope (inVia ${ }^{\mathrm{TM}}$ Reflex, Renishaw) equipped with a 785 $\mathrm{nm}$ diode laser (nominal output $300 \mathrm{~mW}$ ) as excitation source, 1200 grooves $/ \mathrm{mm}$ diffraction grating and a front-illuminated Peltier-cooled CCD camera (1024 pixel x 512 pixel). SERS spectra were measured using a 100x objective with an N.A. of 0.9, a laser power of $1.7 \mathrm{~mW}$ (at the surface), and an integration time of $1 \mathrm{~s}$. For data analysis, 100 spectra were taken from $10 \times 10 \mu \mathrm{m}^{2}$ areas with a distance of $1 \mu \mathrm{m}$ (in $\mathrm{x}$ and $\mathrm{y}$ ) between each point and averaged afterwards. To check the homogeneity of the assemblies the measurements were repeated at least at three positions spaced at $\mathrm{mm}$ intervals and randomly distributed over the substrate for each sample. In the same manner, the batchto-batch reproducibility was also probed for at least two sample batches of each lattice parameter. For lattice parameter-dependent SERS plots, the averaged $1081 \mathrm{~cm}^{-1}$ signals were fitted and the intensities plotted after baseline subtraction using the WIRE $^{\mathrm{TM}} 4$ software package.

Acknowledgements. The authors thank Dr. Guillermo González-Rubio for providing nanoparticles and assistance with the synthesis. C.H. acknowledges funding from the Alexander von Humboldt Foundation through a Feodor Lynen fellowship. Funding by the Spanish Ministerio de Economía, Industria y Competitividad (MINECO) is gratefully acknowledged (Grant SEV-2015-0496 in the framework of the Spanish Severo Ochoa Centre of Excellence program, and Grants MAT2016-79053-P and MAT2017-86659-R). A.M. is grateful to the funding from the European Research Council (ERC) under the European Union's Horizon 2020 research and innovation program (grant agreement No. 637116, ENLIGHTMENT). 
Supporting Information Available. Supporting Information is available online free of charge: Further insight on the optimization of nanoparticle assembly including structural characterization of the PDMS mold; details of the theoretical calculations (near field intensity, enhancement factor, Rayleigh anomalies); details on SERS measurements and calculation of normalized signal intensity per particle and random layer characterization. 


\section{References}

(1) Fedick, P. W.; Bills, B. J.; Manicke, N. E.; Cooks, R. G. Forensic Sampling and Analysis from a Single Substrate: Surface-Enhanced Raman Spectroscopy Followed by Paper Spray Mass Spectrometry. Anal. Chem. 2017, 89, 1097310979.

(2) Baumberg, J.; Bell, S.; Bonifacio, A.; Chikkaraddy, R.; Chisanga, M.; Corsetti, S.; Delfino, I.; Eremina, O.; Fasolato, C.; Faulds, K.; Fleming, H.; Goodacre, R.; Graham, D.; Hardy, M.; Jamieson, L.; Keyes, T.; Królikowska, A.; Kuttner, C.; Langer, J.; et al. SERS in Biology/biomedical SERS: General Discussion. Faraday Discuss. 2017, 205, 429-456.

(3) Premasiri, W. R.; Chen, Y.; Fore, J.; Brodeur, A.; Ziegler, L. D. SERS Biomedical Applications: Diagnostics, Forensics, and Metabolomics. Front. Adv. Mol. Spectrosc. 2017, 327-367.

(4) Sun, F.; Hung, H.-C.; Sinclair, A.; Zhang, P.; Bai, T.; Galvan, D. D.; Jain, P.; Li, B.; Jiang, S.; Yu, Q. Hierarchical Zwitterionic Modification of a SERS Substrate Enables Real-Time Drug Monitoring in Blood Plasma. Nat. Commun. 2016, 7, 13437.

(5) Graham, D.; Goodacre, R.; Arnolds, H.; Masson, J.-F.; Schatz, G.; Baumberg, J.; Kim, D.-H.; Aizpurua, J.; Lum, W.; Silvestri, A.; de Nijs, B.; Xu, Y.; Di Martino, G.; Natan, M.; Schlücker, S.; Wuytens, P.; Bruzas, I.; Kuttner, C.; Hardy, M.; et al. Theory of SERS Enhancement: General Discussion. Faraday Discuss. 2017, 205, 173-211.

(6) Jeanmaire, D. L.; Van Duyne, R. P. Surface Raman Spectroelectrochemistry: Part I. Heterocyclic, Aromatic, and Aliphatic Amines Adsorbed on the Anodized Silver Electrode. J. Electroanal. Chem. Interfacial Electrochem. 1977, 84, 1-20.

(7) Albrecht, M. G.; Creighton, J. A. Anomalously Intense Raman Spectra of Pyridine at a Silver Electrode. J. Am. Chem. Soc. 1977, 99, 5215-5217.

(8) Moskovits, M. Persistent Misconceptions Regarding SERS. Phys. Chem. Chem. Phys. 2013, 15, 5301-5311.

(9) Jahn, M.; Patze, S.; Hidi, I. J.; Knipper, R.; Radu, A. I.; Mühlig, A.; Yüksel, S.; Peksa, V.; Weber, K.; Mayerhöfer, T.; Cialla-May, D.; Popp, J. Plasmonic Nanostructures for Surface Enhanced Spectroscopic Methods. Analyst 2016, 141, 756-793.

(10) Di Martino, G.; Turek, V. A.; Tserkezis, C.; Lombardi, A.; Kuhn, A.; Baumberg, J. J. Plasmonic Response and SERS Modulation in Electrochemical Applied Potentials. Faraday Discuss. 2017, 205, 537-545.

(11) Luo, S.-C.; Sivashanmugan, K.; Liao, J.-D.; Yao, C.-K.; Peng, H.-C. Nanofabricated SERS-Active Substrates for Single-Molecule to Virus Detection in Vitro: A Review. Biosens. Bioelectron. 2014, 61, 232-240.

(12) Willets, K. A.; Van Duyne, R. P. Localized Surface Plasmon Resonance Spectroscopy and Sensing. Annu. Rev. Phys. Chem 2007, 58, 267-297. 
(13) Yang, A.; Hoang, T. B.; Dridi, M.; Deeb, C.; Mikkelsen, M. H.; Schatz, G. C.; Odom, T. W. Real-Time Tunable Lasing from Plasmonic Nanocavity Arrays. Nat. Commun. 2015, 6.

(14) Cha, H.; Yoon, J. H.; Yoon, S. Probing Quantum Plasmon Coupling Using Gold Nanoparticle Dimers with Tunable Interparticle Distances Down to the Subnanometer Range. ACS Nano 2014, 8, 8554-8563.

(15) Hao, E.; Schatz, G. C. Electromagnetic Fields around Silver Nanoparticles and Dimers Shape Effects in Plasmon Resonance of Individual Colloidal Silver Nanoparticles Experimental Observation of Narrow Surface Plasmon Resonances in Gold Nanoparticle Arrays Electromagnetic Fields. J. Chem. Phys. 2004, 120, 11101-10871.

(16) Huang, Y.; Zhou, Q.; Hou, M.; Ma, L.; Zhang, Z. Nanogap Effects on near-and Far-Field Plasmonic Behaviors of Metallic Nanoparticle Dimers. Phys. Chem. Chem. Phys. 2015, 17, 29293-29298.

(17) Mosier-Boss, P. A. Review of SERS Substrates for Chemical Sensing. Nanomaterials 2017, 7, 142 (1-30).

(18) Strobbia, P.; Languirand, E.; Cullum, B. M. Recent Advances in Plasmonic Nanostructures for Sensing: A Review. Opt. Eng. 2015, 54, 100902.

(19) Kraus, T.; Malaquin, L.; Schmid, H.; Riess, W.; Spencer, N. D.; Wolf, H. Nanoparticle Printing with Single-Particle Resolution. Nat. Nanotechnol. 2007, 2, $570-576$.

(20) Hanske, C.; Müller, M. B.; Bieber, V.; Tebbe, M.; Jessl, S.; Wittemann, A.; Fery, A. The Role of Substrate Wettability in Nanoparticle Transfer from Wrinkled Elastomers: Fundamentals and Application toward Hierarchical Patterning. Langmuir 2012, 28, 16745-16750.

(21) Volk, K.; Fitzgerald, J. P. S.; Ruckdeschel, P.; Retsch, M.; König, T. A. F.; Karg, M. Reversible Tuning of Visible Wavelength Surface Lattice Resonances in SelfAssembled Hybrid Monolayers. Adv. Opt. Mater. 2017, 5, 1600971.

(22) Greybush, N. J.; Liberal, I.; Malassis, L.; Kikkawa, J. M.; Engheta, N.; Murray, C. B.; Kagan, C. R. Plasmon Resonances in Self-Assembled Two-Dimensional Au Nanocrystal Metamolecules. ACS Nano 2017, 11, 2917-2927.

(23) Gu, X.; Tian, S.; Zhou, Q.; Adkins, J.; Gu, Z.; Li, X.; Zheng, J. SERS Detection of Polycyclic Aromatic Hydrocarbons on a Bowl-Shaped Silver Cavity Substrate. RSC Adv. 2013, 3, 25989.

(24) Polavarapu, L.; Liz-Marzán, L. M. Towards Low-Cost Flexible Substrates for Nanoplasmonic Sensing. Phys. Chem. Chem. Phys. 2013, 15, 5288.

Bodelón, G.; Montes-García, V.; López-Puente, V.; Hill, E. H.; Hamon, C.; Sanz-Ortiz, M. N.; Rodal-Cedeira, S.; Costas, C.; Celiksoy, S.; Pérez-Juste, I.; Scarabelli, L.; La Porta, A.; Pérez-Juste, J.; Pastoriza-Santos, I.; Liz-Marzán, L. M. Detection and Imaging of Quorum Sensing in Pseudomonas Aeruginosa Biofilm Communities by Surface-Enhanced Resonance Raman Scattering. Nat. Mater. 2016, 15, 1203-1211. 
(26) Ross, M. B.; Mirkin, C. A.; Schatz, G. C. Optical Properties of One-, Two-, and Three-Dimensional Arrays of Plasmonic Nanostructures. J. Phys. Chem. C 2016, 120, 816-830.

(27) Hamon, C.; Novikov, S. M.; Scarabelli, L.; Solís, D. M.; Altantzis, T.; Bals, S.; Taboada, J. M.; Obelleiro, F.; Liz-Marzán, L. M. Collective Plasmonic Properties in Few-Layer Gold Nanorod Supercrystals. ACS Photonics 2015, 2, 1482-1488.

(28) Hanske, C.; Tebbe, M.; Kuttner, C.; Bieber, V.; Tsukruk, V. V; Chanana, M.; König, T. A. F.; Fery, A. Strongly Coupled Plasmonic Modes on Macroscopic Areas via Template-Assisted Colloidal Self-Assembly. Nano Lett. 2014, 14, $6863-6871$.

(29) Hentschel, M.; Dregely, D.; Vogelgesang, R.; Giessen, H.; Liu, N. Plasmonic Oligomers: The Role of Individual Particles in Collective Behavior. ACS Nano 2011, 5, 2042-2050.

(30) Wang, D.; Yang, A.; Wang, W.; Hua, Y.; Schaller, R. D.; Schatz, G. C.; Odom, T. W. Band-Edge Engineering for Controlled Multi-Modal Nanolasing in Plasmonic Superlattices. Nat. Nanotechnol. 2017, 12, 889-894.

(31) Yan, B.; Thubagere, A.; Premasiri, W. R.; Ziegler, L. D.; Negro, L. D.; Reinhard, B. M. Engineered SERS Substrates with Multiscale Signal Enhancement: Nanoparticle Cluster Arrays. ACS Nano 2009, 3, 1190-1202.

(32) Yan, B.; Boriskina, S. V.; Reinhard, B. M. Design and Implementation of Noble Metal Nanoparticle Cluster Arrays for Plasmon Enhanced Biosensing. J. Phys. Chem. C 2011, 115, 24437-24453.

(33) Wang, D.; Yang, A.; Hryn, A. J.; Schatz, G. C.; Odom, T. W. Superlattice Plasmons in Hierarchical Au Nanoparticle Arrays. ACS Photonics 2015, 2, 1789 1794.

(34) Hamon, C.; Sanz-Ortiz, M. N.; Modin, E.; Hill, E. H.; Scarabelli, L.; Chuvilin, A.; Liz-Marzán, L. M. Hierarchical Organization and Molecular Diffusion in Gold Nanorod/silica Supercrystal Nanocomposites. Nanoscale 2016, 8, 7914 7922.

(35) Hamon, C.; Novikov, S.; Scarabelli, L.; Basabe-Desmonts, L.; Liz-Marzán, L. M. Hierarchical Self-Assembly of Gold Nanoparticles into Patterned Plasmonic Nanostructures. ACS Nano 2014, 8, 10694-10703.

(36) Macias, G.; Alba, M.; Marsal, L. F.; Mihi, A. Surface Roughness Boosts the SERS Performance of Imprinted Plasmonic Architectures. J. Mater. Chem. C 2016, 4, 3970-3975.

(37) Hanske, C.; González-Rubio, G.; Hamon, C.; Formentín, P.; Modin, E.; Chuvilin, A.; Guerrero-Martínez, A.; Marsal, L. F.; Liz-Marzán, L. M. Large-Scale Plasmonic Pyramidal Supercrystals via Templated Self-Assembly of Monodisperse Gold Nanospheres. J. Phys. Chem. C 2017, 121, 10899-10906.

(38) Yoon, J. H.; Selbach, F.; Langolf, L.; Schlücker, S. Ideal Dimers of Gold Nanospheres for Precision Plasmonics: Synthesis and Characterization at the Single-Particle Level for Identification of Higher Order Modes. Small 2018, 14, 
1702754.

(39) Bao, K.; Mirin, N. A.; Nordlander, P. Fano Resonances in Planar Silver Nanosphere Clusters. Appl. Phys. A 2010, 100, 333-339.

(40) Guo, R.; Hakala, T. K.; Törmä, P. Geometry Dependence of Surface Lattice Resonances in Plasmonic Nanoparticle Arrays. Phys. Rev. B 2017, 95, 155423.

(41) Khlopin, D.; Laux, F.; Wardley, W. P.; Martin, J.; Wurtz, G. A.; Plain, J.; Bonod, N.; Zayats, A. V.; Dickson, W.; Gérard, D. Lattice Modes and Plasmonic Linewidth Engineering in Gold and Aluminum Nanoparticle Arrays. J. Opt. Soc. Am. B 2017, 34, 691-700.

(42) Auguié, B.; Bendaña, X. M.; Barnes, W. L.; García de Abajo, F. J. Diffractive Arrays of Gold Nanoparticles near an Interface: Critical Role of the Substrate. Phys. Rev. B 2010, 82, 155447.

(43) Le Ru, E.; Etchegoin, P. Principles of Surfance Enhanced Raman Spectroscopy and Related Plasmonic Effets. Elsevier 2009.

(44) Huang, Y.; Zhang, X.; Ringe, E.; Hou, M.; Ma, L.; Zhang, Z. Tunable Lattice Coupling of Multipole Plasmon Modes and Near-Field Enhancement in Closely Spaced Gold Nanorod Arrays. Sci. Rep. 2016, 6, 23159.

(45) Zhang, Y.; Li, X.; Xue, B.; Kong, X.; Liu, X.; Tu, L.; Chang, Y. A Facile and General Route to Synthesize Silica-Coated SERS Tags with the Enhanced Signal Intensity. Sci. Rep. 2015, 5, 14934.

(46) Rodríguez-Lorenzo, L.; Álvarez-Puebla, R. A.; García de Abajo, F. J.; LizMarzán, L. M. Surface Enhanced Raman Scattering Using Star-Shaped Gold Colloidal Nanoparticles. J. Phys. Chem. C 2010, 114, 7336-7340.

(47) Scarabelli, L.; Sánchez-Iglesias, A.; Pérez-Juste, J.; Liz-Marzán, L. M. A “Tips and Tricks" Practical Guide to the Synthesis of Gold Nanorods. J. Phys. Chem. Lett. 2015, 6, 4270-4279.

(48) Xia, Y.; Rogers, J. A.; Paul, K. E.; Whitesides, G. M. Unconventional Methods for Fabricating and Patterning Nanostructures. Chem. Rev. 1999, 99, 1823-1848.

(49) Qin, D.; Xia, Y.; Whitesides, G. M. Soft Lithography for Micro- and Nanoscale Patterning. Nat. Protoc. 2010, 5, 491-502.

(50) Johnson, P. B.; Christy, R. W. Optical Constants of the Noble Metals. Phys. Rev. $B$ 1972, 6, 4370-4379. 


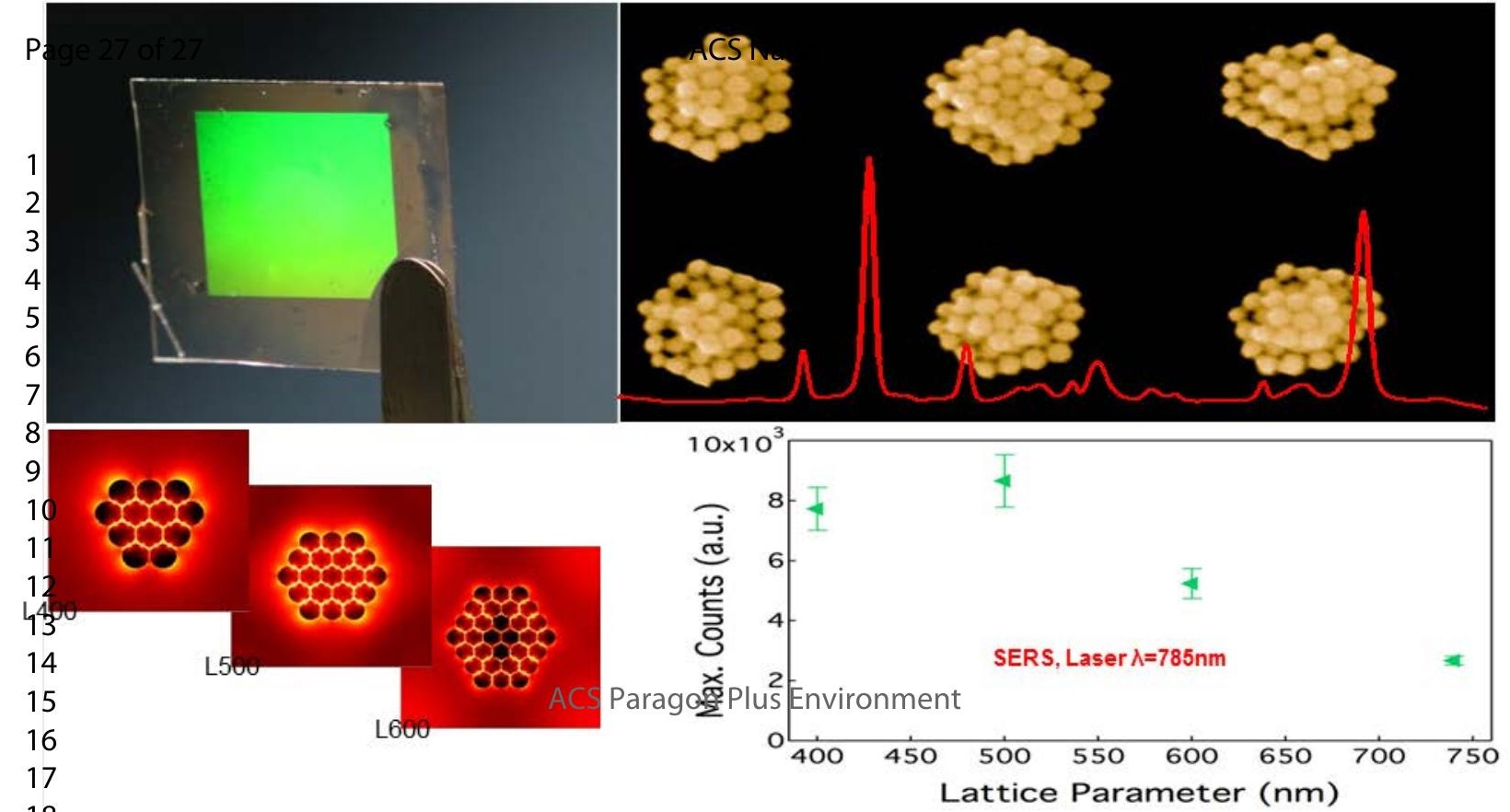

